Quim. Nova, Vol. 34, No. 3, 444-449, 2011

\title{
DETERMINAÇÃO DE BIFENILOS POLICLORADOS EM SORO DE CORDÃO UMBILICAL ATRAVÉS DE EXTRAÇÃO POR HIDRÓLISE ÁCIDA SEGUIDA DE CROMATOGRAFIA A GÁS ACOPLADA A UM MICRODETECTOR DE CAPTURA DE ELÉTRONS
}

\author{
Susana Mohr, Thiago Guilherme Schwanz e Roger Wagner \\ Departamento de Tecnologia e Ciência dos Alimentos, Centro de Ciências Rurais, Universidade Federal de Santa Maria, \\ Av. Roraima, 1000, 97119-900 Santa Maria - RS, Brasil \\ Leandra Soldatelli e Ijoni Hilda Costabeber* \\ Departamento de Morfologia, Centro de Ciências da Saúde, Universidade Federal de Santa Maria, Av. Roraima, 1000, 97105-900 \\ Santa Maria - RS, Brasil
}

Recebido em 9/6/10; aceito em 17/10/10; publicado na web em 26/1/11

\begin{abstract}
DETERMINATION OF POLYCHLORINATED BIPHENYLS IN UMBILICAL CORD SERUM BY ACID HYDROLYZE EXTRACTION FOLLOWED BY GAS CHROMATOGRAPHY WITH MICRO-ELECTRON-CAPTURE DETECTION. The present work describes the determination of polychlorinated biphenyls in 123 umbilical cord serum samples by liquid-liquid extraction method with acid hydrolyze step and analysis by GC- $\mu \mathrm{ECD}$. The analytical method was evaluated with following figures of merit for all PCBs: linearity $(>0.997)$; precision $(<12.55 \%)$; recoveries $(73-119 \%)$; limit of detection $\left(0.1 \mathrm{ng} \mathrm{mL}^{-1}\right)$; limit of quantification $\left(0.25-0.5 \mathrm{ng} \mathrm{mL}^{-1}\right)$. The results showed high contamination in the analyzed samples. PCB more frequent was 138 $(66.7 \%)$, followed by $180(55.3 \%)$ and $52(51.3 \%)$.
\end{abstract}

Keywords: PCBs; umbilical cord; gas chromatography.

\section{INTRODUÇÃO}

Os bifenilos policlorados (PCBs) pertencem à classe de compostos orgânicos persistentes, presentes em inúmeras e complexas amostras indicadoras da contaminação ambiental. Foram descobertos há mais de 100 anos, tendo a sua utilização pela indústria reconhecida precocemente devido às suas propriedades físicas. ${ }^{1}$ Sua estrutura molecular tem uma considerável resistência a ácidos, bases, altas temperaturas e correntes elétricas, além de ser uma substância não inflamável. ${ }^{2}$ São praticamente insolúveis em água e facilmente solúveis em carboidratos, gorduras e outros compostos orgânicos, sendo rapidamente absorvidos por tecidos gordurosos. Inicialmente detectados no meio ambiente no final dos anos 60 , em seguida foram identificados como contaminantes em quase todos os componentes do ecossistema global, incluindo ar, água, solo, peixes, animais, plantas, sangue humano, tecidos adiposos e leite. ${ }^{1}$ A utilização dos PCBs em diversos processos industriais originou uma ampla contaminação do meio ambiente, e, consequentemente, de todos os alimentos que fazem parte da cadeia alimentar. Uma vez no meio ambiente, os PCBs seguem um caminho diferente de dispersão e de bioacumulação, segundo as propriedades intrínsecas de cada congênere e do meio em que se encontram. A grande afinidade dos PCBs pela matéria orgânica e sedimentos faz com que sejam adsorvidos facilmente na superfície das partículas, sendo levados até os rios, mares e oceanos. ${ }^{3,4}$ As proporções da dispersão e da transferência destes compostos para os animais, vegetais e seres humanos são difíceis de serem estimadas. ${ }^{1}$

Apesar de sua proibição em nosso país, ocorrida em 1981, equipamentos eletroeletrônicos antigos que os contêm ainda podem ser utilizados até a sua substituição. Em consequência disso, casos de contaminação ambiental e intoxicação são constantemente relatados pelos meios de comunicação, resultado de incidentes em várias regiões.

\footnotetext{
*e-mail: ijonicostabeber@gmail.com
}

Recentemente, em Nova Iguaçú, RJ, foram apreendidos 200.000 L de Ascarel ${ }^{\circledR}$ (denominação comercial do óleo de PCBs no Brasil) em um depósito clandestino da Company Eletric. No local, foram encontrados quatro funcionários contaminados que trabalhavam de vigias, queixandose de fortes dores de cabeça, fraqueza e falta de mobilidade nas mãos. ${ }^{5}$

Como consequência de sua alta persistência e alta tendência à bioacumulação, os PCBs continuam a ser detectados rotineiramente em amostras de tecido humano e animal. O soro e o plasma sanguíneo são as amostras mais frequentemente utilizadas para quantificar os biomarcadores da exposição humana aos poluentes orgânicos persistentes, como os PCBs. ${ }^{6}$ Estudos demonstram que sua concentração no soro aumenta conforme a idade, devido ao seu grande poder de bioacumulação..$^{7-12}$ Além da idade, o aumento de sua concentração também pode estar associado com o hábito de fumar e com o fato de residir em áreas de maior risco de contaminação. ${ }^{13-15}$

A concentração de PCBs em plasma sanguíneo de cordão umbilical é um ótimo indicador da exposição pré-natal, ${ }^{16}$ pois os níveis de resíduos detectados no soro materno e no soro de cordão umbilical demonstram uma significante relação, confirmando sua eficiente transferência transplacentária. ${ }^{16-18}$ Assim que entram no sistema sanguíneo fetal, os PCBs causam riscos à saúde dos fetos e recémnascidos, os quais são reconhecidamente muito mais vulneráveis aos efeitos dos poluentes ambientais. ${ }^{19,20} \mathrm{~A}$ exposição do feto ainda no útero pode prejudicar o seu crescimento, causando efeitos adversos no peso ao nascer, comprimento e perímetro cefálico, e também à sua saúde, com alterações no escore do Apgar. ${ }^{20}$

Embora alguns estudos recentes tenham constatado a redução no nível de PCBs em matrizes biológicas, ${ }^{17} \mathrm{em}$ especial no sangue, estes ainda continuam a ser detectados com bastante frequência. $\mathrm{O}$ maior número de pesquisas encontra-se nos países que possuíam fábricas de PCBs, como Itália, Alemanha, Japão, Estados Unidos, entre outros. Em geral, os congêneres 138, 153 e 180 são os que possuem as 
maiores concentrações de resíduos detectadas., ${ }^{8,14,15,17,21,22}$ Estes três congêneres, juntamente com os congêneres 28, 52, 101 e 118 são denominados "os sete indicadores", responsáveis pela maior parte da contaminação ambiental.

A análise cromatográfica de contaminantes em matrizes biológicas geralmente necessita de pré-tratamento para limpeza e concentração dos analitos. As razões para isso são muitas, mas destacam-se a complexidade da amostra, marcada pela existência de substâncias não voláteis incompatíveis com as colunas cromatográficas e a concentração em nível de traços das substâncias a serem analisadas. ${ }^{23} \mathrm{O}$ sangue é constituído de elementos celulares (hemácias, leucócitos e plaquetas) e plasma (água, proteínas, gorduras, carboidratos, eletrólitos, sais orgânicos e minerais, e hormônios). Dentre as proteínas contidas no plasma estão a albumina, a globulina e o fibrinogênio, sendo esta última fundamental no processo de coagulação do sangue. ${ }^{24}$ O soro possui a mesma composição do plasma, com exceção da presença do fibrinogênio, apresentando em média $2,4 \mathrm{~g} \mathrm{~L}^{-1}$ de gordura, com variações de 1,7 a $3,4 \mathrm{~g} \mathrm{~L}^{-1} \cdot{ }^{17} \mathrm{O}$ teor de gordura é importante para a determinação de PCBs, pois estes compostos são lipofílicos e encontram-se dispersos nos glóbulos de gordura.

Diferentes técnicas de extração têm sido empregadas na análise de PCBs em soro sanguíneo, como a extração em fase sólida (SPE = Solid Phase Extraction), ${ }^{25-27}$ a microextração em fase sólida (SPME = Solid Phase Micro Extraction) $)^{28,29}$ e a extração líquido-líquido (LLE = Liquid-Liquid Extraction). ${ }^{30-33}$ A extração líquido-líquido destacase pelo grande número de aplicações, onde a eficiência da extração depende da afinidade do soluto pelo solvente de extração, da razão das fases e do número de extrações. Esta técnica apresenta algumas vantagens, como simplicidade e grande número de fases extratoras disponíveis, as quais fornecem uma ampla faixa de solubilidade e seletividade. Adicionalmente, o emprego da hidrólise ácida junto à extração líquido-líquido destrói um grande número de compostos interferentes que podem estar presentes na fase orgânica, diminuindo a contaminação na coluna cromatográfica. ${ }^{34}$

A determinação dos PCBs normalmente é realizada empregandose a cromatografia a gás acoplada aos detectores de captura de elétrons, ${ }^{14,16,30,33,35}$ espectrometria de massas ${ }^{8,10,17,18,20,31}$ ou ambos. ${ }^{11,12,19,27} \mathrm{~A}$ validação do método analítico é de extrema importância para garantir que este gere informações confiáveis e interpretáveis sobre a amostra,${ }^{36}$ diminuindo ou controlando os fatores que levam à imprecisão de um dado gerado. Os parâmetros a serem validados variam em função do tipo de procedimento, de acordo com as características a serem estudadas e o método a aplicar. Os conceitos mais importantes para validação do método analítico em geral são: linearidade, sensibilidade, precisão, exatidão, recuperação, repetibilidade, reprodutibilidade, especificidade, limite de detecção e limite de quantificação. ${ }^{37}$

O objetivo do presente estudo foi validar um método de extração simples e rápido, envolvendo a partição da amostra com solvente orgânico e hidrólise ácida da matriz, seguido de análise através de cromatógrafo a gás equipado com microdetector de captura de elétrons $(\mathrm{GC}-\mu \mathrm{ECD}=$ Gas Chromatography $-\mu$ Electron Capture Detection) e confirmação através de cromatógrafo a gás acoplado à espectrometria de massas (GC/MS = Gas Chromatography/Mass Spectrometry), para determinar a concentração de $\mathrm{PCBs}$ indicadores de contaminação ambiental em amostras de soro de cordão umbilical.

\section{PARTE EXPERIMENTAL}

\section{Materiais}

\section{Amostras}

Para a realização do presente estudo foram coletadas 123 amostras de sangue arterial de cordão umbilical de recém-nascidos de mães que tiveram seus filhos no Centro Obstétrico do Hospital Universitário de Santa Maria (HUSM), durante o ano de 2006. O grupo das doadoras era constituído de mulheres que residiam na região central do Rio Grande do Sul, com idade média de 25 anos, sendo a maioria casada, do lar e não fumantes. A coleta de sangue foi efetuada no segmento de cordão umbilical, junto à placenta, logo após a ligadura e secção do mesmo. Para a determinação dos congêneres de PCBs utilizou-se o soro, obtido por centrifugação do sangue. Após a centrifugação, as amostras foram armazenadas até o momento das análises a uma temperatura de $-20^{\circ} \mathrm{C}$. $\mathrm{O}$ volume aproximado das amostras de soro de cordão umbilical foi de $0,5 \mathrm{~mL}$. Devido ao baixo volume de amostra, não foi possível realizar a análise dos compostos na gordura do soro. O protocolo aplicado foi previamente aprovado pelo Comitê de Ética em Pesquisa da UFSM através da Carta de Aprovação número 0014.0.246.000-06.

\section{Preparação dos materiais}

Todos os materiais foram cuidadosamente limpos a fim de minimizar uma possível contaminação. Inicialmente foram lavados com água e detergente neutro, enxaguados três vezes com água destilada e secos em estufa. Após, foram enxaguados três vezes com acetona e hexano de forma alternada e, finalmente, submetidos a $150{ }^{\circ} \mathrm{C}$ durante $12 \mathrm{~h}{ }^{38}$

\section{Padrões}

Uma solução estoque de PCBs contendo $10 \mu \mathrm{g} \mathrm{mL} \mathrm{m}^{-1}$ dos congêneres 28 (2,4,4'-triclorobifenil), 52 (2,2',5,5'-tetraclorobifenil), 138 (2,2',3,4,4',5'-hexaclorobifenil), 153 (2,2',4,4',5,5'-hexaclorobifenil) e 180 (2,2',3,4,4',5,5'- heptaclorobifenil) foi utilizada na identificação e nas etapas de validação. Os padrões foram adquiridos de Supelco, Inc., Bellefonte, Pensilvania, USA, com certificado de pureza superior a $99 \%$.

\section{Reagentes}

O solvente utilizado nas extrações e ensaios cromatográficos foi $n$-hexano Nanograde ${ }^{\circledR}$ com certificação para análises de resíduos de pesticidas (Mallinckrodt Baker, Inc., NJ, USA). Foram também utilizados ácido sulfúrico PA (Vetec Química Fina Ltda, Duque de Caxias, RJ, Brasil) e nitrogênio grau 5,0 analítico (White Martins, Praxair Inc.).

\section{Métodos}

\section{Extração dos PCBs}

O método de extração utilizado segue estudo de Otero et al., ${ }^{34}$ com algumas modificações. Em um tubo de ensaio foi inicialmente adicionado 0,5 mL de soro, seguido dos padrões dos PCBs 28, 52, 138, 153 e 180, e agitação em vortex (1 min). A digestão ácida foi realizada através da adição de $3 \mathrm{~mL}$ de $n$-hexano e $2 \mathrm{~mL}$ de ácido sulfúrico. A mistura foi, então, levada novamente ao vortex e, em seguida, à centrífuga (10 min a $2.000 \mathrm{rpm})$. Após, a fase de $n$-hexano foi retirada com o auxílio de uma pipeta de Pasteur e o conteúdo restante (soro + ácido) foi re-extraído por mais duas vezes com 2 $\mathrm{mL}$ de $n$-hexano. No tubo em que foram coletados os extratos de $n$ hexano foram adicionados $2 \mathrm{~mL}$ de ácido sulfúrico e a mistura, após agitação, foi centrifugada. Em seguida, a fase orgânica foi separada e transferida para um tubo coletor, onde foi levada para sua evaporação total no aparelho Mini-Vap (Supelco Inc., Bellefonte, Pensilvania, USA), com o auxílio de gás nitrogênio. Terminado o procedimento de evaporação, a amostra foi ressuspendida em $0,5 \mathrm{~mL}$ de $n$-hexano e então submetida à análise cromatográfica.

\section{Análise cromatográfica}

Os extratos obtidos foram analisados em um cromatógrafo a gás equipado com microdetector de captura de elétrons (GC- $\mu \mathrm{ECD})$ de 
$\mathrm{Ni}^{63}$, marca Agilent, modelo 6890 Plus. Um microlitro dos extratos foi introduzido em um injetor do tipo split/splitless no modo splitless, onde permaneceu neste modo por 1 min. Logo após a válvula foi aberta com divisão de fluxo de 1:20. A temperatura do injetor foi constante de $280^{\circ} \mathrm{C}$. A separação dos analitos foi realizada em uma coluna capilar de sílica fundida HP-5, marca J\&W Scientific, com $30 \mathrm{~m}$ de comprimento, $0,32 \mathrm{~mm}$ de diâmetro interno e $0,25 \mu \mathrm{m}$ de espessura de fase estacionária. O nitrogênio foi utilizado como gás de arraste sob pressão constante de 7,2 psi, fornecendo uma vazão inicial de $1,5 \mathrm{~mL} \mathrm{~min}^{-1}$. A programação da temperatura do forno foi: $60{ }^{\circ} \mathrm{C}(2 \mathrm{~min})$, taxa de aquecimento a $30^{\circ} \mathrm{C} / \mathrm{min}$ até $190{ }^{\circ} \mathrm{C}(5 \mathrm{~min})$, seguida de taxa de $5{ }^{\circ} \mathrm{C} / \mathrm{min}$ até $220^{\circ} \mathrm{C}$ ( $\left.5 \mathrm{~min}\right)$ e, finalmente, a 20 ${ }^{\circ} \mathrm{C} / \mathrm{min}$ até $300{ }^{\circ} \mathrm{C}(1 \mathrm{~min})$. A temperatura do detector foi mantida a $320^{\circ} \mathrm{C}$. Os dados cromatográficos foram analisados através do programa HP ChemStation. A identificação dos compostos foi realizada pela comparação dos tempos de retenção dos picos encontrados nas amostras com os tempos de retenção individuais dos padrões de bifenilos policlorados em estudo, sob as mesmas condições de trabalho.

A confirmação dos resultados obtidos foi realizada em um cromatógrafo a gás acoplado à espectrometria de massas (GC/MS), marca Agilent, modelo 6890, equipado com injetor automático e com a mesma coluna utilizada no GC- $\mu$ ECD. Este operou no modo de monitoramento de íons selecionados ( $\mathrm{SIM}=$ Single Ion Monitoring) com ionização por impacto de elétrons $(\mathrm{EI}=$ Electron Impact $)$ a $70 \mathrm{eV}$, com a seguinte programação: $60^{\circ} \mathrm{C}(2 \mathrm{~min})$, taxa de aquecimento a 5 ${ }^{\circ} \mathrm{C} / \mathrm{min}$ até $220{ }^{\circ} \mathrm{C}(5 \mathrm{~min})$, seguida de taxa de $20^{\circ} \mathrm{C} / \mathrm{min}$ até $300{ }^{\circ} \mathrm{C}$ ( 2 min). O hélio foi utilizado como gás de arraste com uma pressão de 13,38 psi, uma vazão de $1,5 \mathrm{~mL} \mathrm{~min}^{-1}$.

A análise qualitativa foi realizada pela comparação da abundância dos íons majoritários de cada um dos cinco congêneres, obtidos a partir dos padrões de referência, com os íons encontrados na amostra. Os íons selecionados foram $\mathrm{m} / \mathrm{z}, 186,256$ e 258 (PCB 28); $\mathrm{m} / \mathrm{z}, 220$, 222, 290 e 292 (PCB 52); $\mathrm{m} / z$, 290, 358, 360 e 362 (PCB 153); $\mathrm{m} / \mathrm{z}$ 235, 290, 360 e 362 (PCB 138); m/z 324, 394, 396 e 398 (PCB 180).

\section{Validação do método analítico}

Para avaliar a eficiência do método de extração proposto foram validados os seguintes parâmetros: linearidade, precisão, exatidão, limite de detecção e limite de quantificação. Para minimizar o efeito da matriz, os testes preliminares e a curva de calibração foram feitos em soro coletado de indivíduos voluntários do grupo de pesquisa, enquanto que os demais procedimentos de validação (recuperação, precisão e limites de detecção e quantificação) foram realizados em amostras de soro de cordão umbilical.

Para o estudo da linearidade preparou-se uma série de 5 amostras de soro, fortificadas com os padrões em concentrações crescentes (de 0,25 a $12 \mathrm{ng} \mathrm{mL}^{-1}$ para o PCB 28 e de 0,5 a $20 \mathrm{ng} \mathrm{mL}^{-1}$ para os demais PCBs). Estas amostras compuseram os pontos da curva e foram preparadas em triplicata, extraídas em dias diferentes, de forma aleatória, e analisadas em duplicata no sistema cromatográfico. Adicionalmente, foram preparadas amostras testemunhas, que incluem a extração (n = 3) somente da matriz, sem adição do analito, e outra utilizando o mesmo volume de água ultrapura ao invés da matriz. A partir destes dados foi obtida a equação da regressão linear e o coeficiente de determinação para cada PCB estudado.

A precisão foi avaliada utilizando-se a estimativa do coeficiente de variação $(\mathrm{CV})$ para a repetibilidade (intradia) e precisão intermediária (interdia). Para a repetibilidade foram preparadas 5 amostras de soro de cordão umbilical iguais, todas fortificadas com $5 \mathrm{ng} \mathrm{mL}^{-1} \mathrm{de}$ cada um dos compostos em estudo. Estas amostras foram analisadas segundo o método proposto, desde a extração (todas no mesmo dia) até a análise cromatográfica. Para a precisão intermediária, foram efetuados os mesmos procedimentos realizados para a análise da repetibilidade, porém em dias diferentes.

A exatidão do método foi avaliada pela técnica de adição do padrão, onde alíquotas de uma mesma amostra de soro de cordão umbilical foram fortificadas com concentrações que variaram de 5,0 a $20 \mathrm{ng} \mathrm{mL}^{-1}(\mathrm{n}=3)$. A exatidão foi relacionada com porcentagem dos analitos recuperados em cada nível de concentração.

$\mathrm{O}$ valor designado como limite de detecção (LD) para cada PCB foi determinado visualmente pela razão sinal/ruído $\geq 3$, a partir de ensaios com amostras de soro de cordão umbilical fortificadas em concentrações decrescentes a partir de $1 \mathrm{ng} \mathrm{mL} \mathrm{m}^{-1}$. De forma semelhante, o limite de quantificação (LQ) também foi obtido pela visualização da razão sinal/ruído, porém com valor $\geq 10$. O valor encontrado para o LQ de cada PCB foi incluído com o menor ponto da curva de calibração.

\section{RESULTADOS E DISCUSSÃO}

\section{Extração}

O método de extração dos PCBs da matriz por hidrólise ácida e partição com solvente orgânico utilizado demonstrou ser eficiente, levando-se em consideração o pequeno volume de amostra disponível, que foi de $0,5 \mathrm{~mL}$. Volumes maiores de amostra, entre 1 e $3 \mathrm{~mL}$, seriam necessários para a extração em fase sólida. ${ }^{8,15,17,18}$ As duas etapas de adição de ácido sulfúrico minimizaram o aparecimento de compostos interferentes durante a análise cromatográfica, aumentando a vida útil da coluna capilar. Estes resultados estão de acordo com os obtidos anteriormente por outros autores, os quais utilizaram método de extração semelhante ao do presente estudo. ${ }^{30,31,33,34}$

\section{Validação do método}

Na Tabela 1 apresentam-se os resultados obtidos para a linearidade e precisão do método proposto. Para todos os compostos em estudo os coeficientes de correlação foram superiores a 0,99 , dentro dos padrões recomendados pela ANVISA, ${ }^{39}$ IUPAC $^{36}$ e INMETRO.${ }^{40}$ Estes coeficientes também indicam que a resposta do microdetector de captura de elétrons foi linear para todos os compostos estudados, nos intervalos de 0,25 a $12 \mathrm{ng} \mathrm{mL}^{-1}$ para o PCB 28, e de 0,5 a $20 \mathrm{ng}$ $\mathrm{mL}^{-1}$ para os demais PCBs.

Tabela 1. Resultados obtidos nas provas de linearidade e precisão

\begin{tabular}{ccccc}
\hline Composto & $\begin{array}{c}\text { Faixa Linear } \\
(\text { ng mL }\end{array}$ - $)$ & $\begin{array}{c}\text { Coeficiente } \\
\text { de correlação } \\
(r)\end{array}$ & $\begin{array}{c}\text { Repetibilidade } \\
(\mathrm{CV}, \%)^{*}\end{array}$ & $\begin{array}{c}\text { Precisão } \\
\text { intermediária } \\
(\mathrm{CV}, \%)^{*}\end{array}$ \\
\hline PCB 28 & $0,25-12$ & 0,999 & 9,53 & 16,59 \\
PCB 52 & $0,50-20$ & 0,998 & 8,03 & 11,26 \\
PCB 153 & $0,50-20$ & 0,999 & 8,27 & 13,01 \\
PCB 138 & $0,50-20$ & 0,999 & 12,55 & 13,85 \\
PCB 180 & $0,50-20$ & 0,997 & 6,75 & 17,76 \\
\hline
\end{tabular}

$\mathrm{CV}=$ Coeficiente de variação. *Calculado ao nível de $5 \mathrm{ng} \mathrm{mL}^{-1}(n=5)$.

A precisão do método analítico foi medida pelo grau de concordância entre os resultados analíticos obtidos para uma mesma amostra, ${ }^{37}$ utilizando-se a estimativa do coeficiente de variação (CV). Para o método em estudo, avaliou-se a precisão em condições de repetibilidade (intradia) e de precisão intermediária (interdia). Para a repetibilidade, os valores do coeficiente de variação ficaram entre 6,75 e 12,55\%, enquanto que para a precisão intermediária, variaram entre 11,26 e $17,76 \%$. Para métodos de análise de traços, como é o caso da análise de resíduos de PCBs, são aceitos coeficientes de va- 
riação de até $20 \%$, dependendo da complexidade da amostra. ${ }^{36}$ Tanto para repetibilidade, quanto para a precisão intermediária, os valores foram menores do que $20 \%$. Para a exatidão do método analítico foi calculada a porcentagem de recuperação em três níveis de concentração. No método proposto, as recuperações variaram entre 73 e 119\% (Tabela 2), valores dentro dos intervalos aceitáveis de recuperação para análise de resíduos, que estão entre 70 e $120 \%$. $^{36}$

Tabela 2. Resultados obtidos para as provas de recuperação $(n=3)$

\begin{tabular}{lccc}
\hline Composto & $\begin{array}{c}\text { Concentração adicionada } \\
\left(\text { ng mL } \text { mL }^{-1}\right.\end{array}$ & $\begin{array}{c}\text { Recuperação } \\
(\%)\end{array}$ & $\begin{array}{c}\text { CV } \\
(\%)\end{array}$ \\
\hline PCB 28 & 5 & 108 & 19 \\
& 8 & 93 & 6 \\
PCB 52 & 12 & 103 & 15 \\
& 5 & 90 & 18 \\
PCB 153 & 8 & 79 & 3 \\
& 12 & 102 & 14 \\
PCB 138 & 5 & 118 & 22 \\
& 12 & 73 & 19 \\
& 8 & 112 & 16 \\
PCB 180 & 10 & 87 & 14 \\
& 20 & 113 & 24 \\
& 8 & 107 & 15 \\
& 12 & 83 & 14 \\
& 20 & 119 & 22 \\
\hline
\end{tabular}

$\mathrm{CV}=$ Coeficiente de Variação

O limite de detecção para todos os compostos em estudo foi de $0,1 \mathrm{ng} \mathrm{mL} \mathrm{m}^{-1}$, valores iguais ou menores do que os encontrados em estudo prévio, ${ }^{41}$ o qual utilizou método de extração semelhante ao do presente estudo, encontrando valores de $0,1 \mathrm{ng} \mathrm{mL}^{-1}$ para o $\mathrm{PCB}$ 138, de $0,24 \mathrm{ng} \mathrm{mL}^{-1}$ para o PCB 153 e de $0,4 \mathrm{ng} \mathrm{mL}^{-1}$ para o PCB 180. Já os limites de quantificação foram de $0,25 \mathrm{ng} \mathrm{mL}^{-1}$ para o $\mathrm{PCB}$ 28 e de $0,5 \mathrm{ng} \mathrm{mL}^{-1}$ para os demais PCBs, resultados semelhantes ou menores que os encontrados em estudo prévio, ${ }^{41}$ com limites de quantificação de $0,33 \mathrm{ng} \mathrm{mL}^{-1}$ para o PCB 138 , de $0,79 \mathrm{ng} \mathrm{mL}^{-1}$ para o PCB 153 e de $1,3 \mathrm{ng} \mathrm{mL}^{-1}$ para o PCB 180 .

\section{Determinação de PCBs nas amostras de soro de cordão umbilical}

O método validado foi então aplicado para analisar os congêneres de PCBs em 123 amostras de soro de cordão umbilical, com volume de 0,5 mL, obtidas de doadoras que tiveram seus filhos no Hospital Universitário de Santa Maria, na região central do Rio Grande do Sul, Brasil. As Figuras 1 e 2 apresentam cromatogramas de GC- $\mu$ ECD e GC/MS, respectivamente, de uma mesma amostra de soro de cordão umbilical contaminada com os 5 congêneres de PCBs em estudo.

Na Tabela 3 apresentam-se os resultados obtidos. O PCB 138 foi o que obteve a maior frequência, sendo detectado acima do limite de quantificação em $66,7 \%$ das amostras analisadas. Este mesmo congênere também foi o que obteve os maiores valores para a média $\left(2,37 \mathrm{ng} \mathrm{mL}^{-1}\right)$, média geométrica $\left(0,68 \mathrm{ng} \mathrm{mL}^{-1}\right)$ e mediana $(1,04 \mathrm{ng}$ $\mathrm{mL}^{-1}$ ), com valores que variaram de $<\mathrm{LQ}$ até $10,59 \mathrm{ng} \mathrm{mL}^{-1}$. O PCB 180 foi o segundo com maior frequência, sendo detectado em 55,3\% das amostras, com valor de $1,43 \mathrm{ng} \mathrm{mL}^{-1}$ para a média, variando de $<$ LQ a 7,08 $\mathrm{ng} \mathrm{mL}^{-1}$. O PCB 52 teve uma frequência semelhante ao

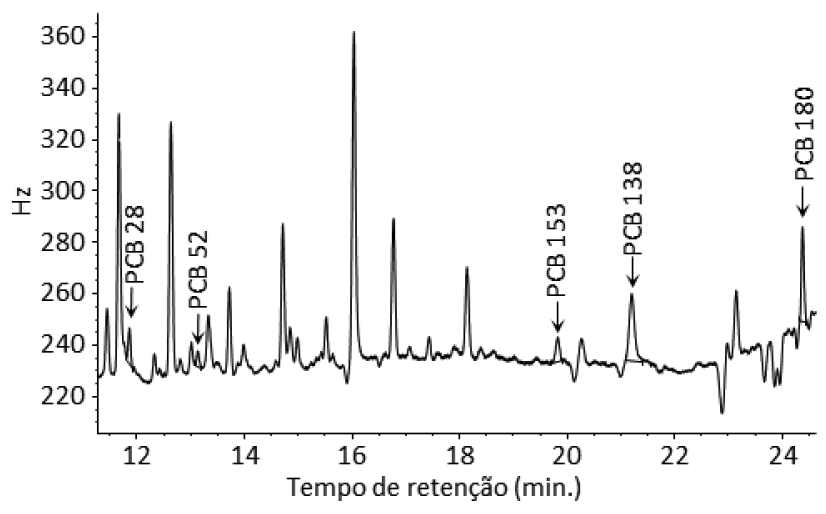

Figura 1. Cromatograma (GC- $\mu E C D)$ de uma amostra de soro de cordão umbilical contaminada, onde: $t_{R}=11,924$ min: $P C B$ 28, $t_{R}=13,194$ min: $P C B$ 52, $t_{R}=19,905: P C B$ 153, $t_{R}=21,296$ min: $P C B 138$ e $t_{R}=24,422: P C B 180$

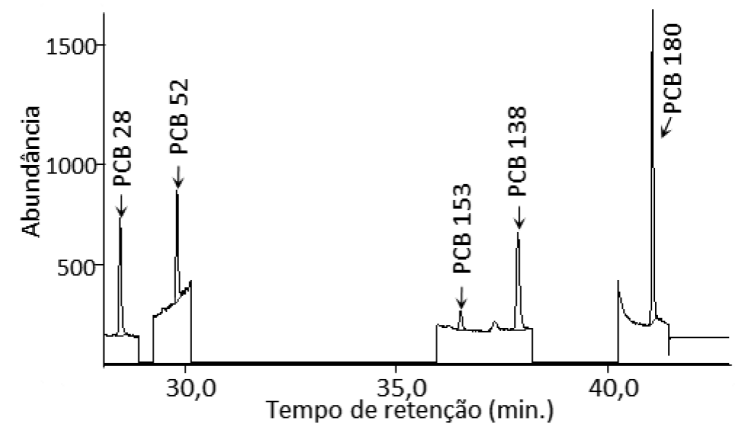

Figura 2. Cromatograma (GC-MS) de uma amostra de soro de cordão umbilical contaminada, onde: $t_{R}=28,325$ min: $P C B$ 28, $t_{R}=29,661$ min: $P C B$ 52, $t_{R}=36,312: P C B$ 153, $t_{R}=37,660$ min: $P C B 138$ e $t_{R}=40,821: P C B 180$

PCB 180 (51,3\%), com média de $0,98 \mathrm{ng} \mathrm{mL}^{-1}$ e valores entre $<$ LQ e $16,93 \mathrm{ng} \mathrm{mL}^{-1}$, seguido do PCB 153 em 48,8\% e do PCB 28, detectado em $16,3 \%$ das amostras analisadas. A concentração média foi de 0,74 $\mathrm{ng} \mathrm{mL} \mathrm{mL}^{-1}$ para o PCB 153, com variações de $<\mathrm{LQ}$ a $5,38 \mathrm{ng} \mathrm{mL}^{-1}$, e de $0,12 \mathrm{ng} \mathrm{mL}^{-1}$ para o PCB 28, com variações de $<$ LQ a $1,17 \mathrm{ng} \mathrm{mL}^{-1}$.

$\mathrm{Na}$ Tabela 4 apresentam-se dados comparativos do presente estudo com dados da literatura, expressos em somatório de PCBs. Como se pode verificar, as concentrações encontradas no presente estudo estão bem acima dos demais, os quais foram realizados em países da Europa. O mais recente, realizado em 2010, na Espanha, ${ }^{30}$ apresentou um valor de $0,61 \mathrm{ng} \mathrm{mL}^{-1}$ de soro de cordão umbilical para o somatório das médias dos PCBs 28, 52, 138, 153 e 180, enquanto que neste estudo o somatório para estes mesmos congêneres de PCBs foi de 5,64 ng mL $\mathrm{mL}^{-1}$. Em outro estudo recente, realizado em 2008, na Eslováquia, ${ }^{22}$ o somatório das médias dos PCBs 138, 153 e 180 foi de $0,99 \mathrm{ng} \mathrm{mL}^{-1}$, enquanto que no presente estudo o somatório para estes três congêneres foi de 4,54 $\mathrm{ng} \mathrm{mL}^{-1}$. Já estudos mais antigos, realizados em 2002, na Bélgica, ${ }^{19}$ e em 1996, na Alemanha, ${ }^{42}$ apresentaram valores menores para o somatório das médias quando comparados aos mais recentes realizados na Europa, já citados anteriormente. Nestes casos, a concentração de PCBs aumentou com o passar dos anos, embora haja estudos constatando sua redução ao longo do tempo. ${ }^{17}$ Esse fato deve-se, provavelmente, ao seu alto poder de bioacumulação. Já em outro estudo recente, realizado em 2009, na Itália, ${ }^{18}$ o valor para o somatório das medianas dos PCBs foi de $0,22 \mathrm{ng} \mathrm{mL}^{-1}$, valor este bem abaixo de outro estudo antigo, realizado em 1996, na Alemanha, ${ }^{43}$ que apresentou valor de $1,82 \mathrm{ng}$ $\mathrm{mL}^{-1}$. Este último foi o que apresentou o valor mais próximo ao do presente estudo, que foi de $2,44 \mathrm{ng} \mathrm{mL}^{-1}$. 
Tabela 3. Frequência de determinação e concentrações dos $\mathrm{PCBs}$ em ng $\mathrm{mL}^{-1}$ (média, desvio-padrão, média geométrica, mediana, mínimo e máximo) em soro de cordão umbilical $(n=123)$

\begin{tabular}{ccccccccc}
\hline Congênere & $n>\mathrm{LQ}$ & $\%>$ LQ & Média & DP & Média geométrica & Mediana & Mínimo & Máximo \\
\hline PCB 28 & 20 & 16,3 & 0,12 & 0,19 & 0,07 & 0,05 & $<$ LQ & 1,17 \\
PCB 52 & 63 & 51,3 & 0,98 & 2,27 & 0,25 & 0,50 & $<$ LQ & 16,93 \\
PCB 153 & 60 & 48,8 & 0,74 & 0,98 & 0,24 & 0,05 & $<\mathrm{LQ}$ & 5,38 \\
PCB 138 & 82 & 66,7 & 2,37 & 2,66 & 0,68 & 1,04 & $<\mathrm{LQ}$ & 10,59 \\
PCB 180 & 68 & 55,3 & 1,43 & 1,63 & 0,40 & 0,80 & $<\mathrm{LQ}$ & 7,08 \\
LPCBs & & & 5,64 & & 1,64 & 2,44 & & \\
\hline
\end{tabular}

$n$ : Número de amostras. DP: Desvio Padrão. Valores abaixo do limite de quantificação (LQ) foram considerados como LD/2 para o cálculo da média e mediana. LD: $0,1 \mathrm{ng} \mathrm{mL}^{-1}$ de soro de cordão umbilical. LQ: $0,25 \mathrm{ng} \mathrm{mL}^{-1}$ de soro de cordão umbilical (PCB 28) e $0,5 \mathrm{ng} \mathrm{mL}^{-1}$ de soro de cordão umbilical (PCBs 52, 153, 138 e 180).

Tabela 4. Concentrações de PCBs $\left(n g \mathrm{~mL}^{-1}\right)$ em soro de cordão umbilical de diferentes países

\begin{tabular}{cccc}
\hline País/Ano & $\mathrm{N}^{\circ}$ amostras & Média & Mediana \\
\hline Brasil, 2010* & 123 & $5,64^{\mathrm{a}}$ & $2,44^{\mathrm{a}}$ \\
Espanha, 2010 $^{30}$ & 410 & $0,61^{\mathrm{a}}$ & - \\
Itália, 2009 $^{18}$ & 70 & - & $0,22^{\mathrm{c}}$ \\
Eslováquia, 2008 $^{22}$ & 1087 & $0,99^{\mathrm{c}}$ & $0,77^{\mathrm{c}}$ \\
Bélgica, 2002 & 44 & $0,47^{\mathrm{b}}$ & - \\
Alemanha, 1996 & 373 & $0,56^{\mathrm{c}}$ & - \\
Alemanha, 1996 & 80 & - & $1,82^{\mathrm{a}}$ \\
\hline
\end{tabular}

* Este estudo. a Somatório dos PCBs 28, 52, 138, 153 e 180. ${ }^{\mathrm{b}}$ Somatório

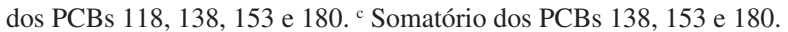

A ATSDR (Agency for Toxic Substances and Disease Registry) ${ }^{44}$ estabelece valores de referência dos PCBs para a população americana. Os dados foram reportados em média geométrica, separados de acordo com a faixa de idade, sexo e raça. Para recém-nascidos não foram estabelecidos valores, entretanto há valores para mulheres, na faixa de 20 anos ou mais, de raça branca ou negra. Considerando o fato de que os níveis de resíduos detectados no soro materno e no soro de cordão umbilical demonstram uma significante relação, devido a sua eficiente transferência transplacentária, ${ }^{16-18}$ pode-se comparar os valores do presente estudo com os que são estabelecidos pela ATSDR para mulheres em idade reprodutiva, ou seja, na faixa de 20 anos ou mais. Somando-se os valores das médias geométricas dos PCBs 28 , $52,138,153$ e 180 tem-se o valor de referência $0,34 \mathrm{ng} \mathrm{mL}^{-1}$, bem abaixo do que foi detectado no presente estudo, de 1,64 $\mathrm{ng} \mathrm{mL}^{-1}$.

Estudos investigando a contaminação de alimentos provenientes do mesmo estado foram realizados anteriormente e constataram a presença de contaminação em leite, queijos e produtos cárneos. O somatório das médias dos PCBs 28, 52, 138, 153 e 180 foi de $24,85 \mathrm{ng} \mathrm{g}^{-1}$ de gordura para queijos, ${ }^{45}$ de $15,5 \mathrm{ng} \mathrm{g}^{-1}$ de gordura para leites pasteurizados ${ }^{46} \mathrm{e}$ de $31,19 \mathrm{ng} \mathrm{g}^{-1}$ de gordura para salsichas hot $\operatorname{dog} .{ }^{47}$ Segundo a legislação brasileira, estabelecida pelo Ministério da Agricultura, Pecuária e Abastecimento, ${ }^{48}$ estes valores estão abaixo dos limites máximos estabelecidos, não apresentando, portanto, indicações de riscos à saúde com o consumo destes alimentos. Apesar disso, a contaminação nos alimentos está presente, assim como constatada nas amostras de soro de cordão umbilical da mesma região. Como este é o primeiro estudo a analisar e demonstrar diretamente os níveis de PCB em soro de cordão umbilical de recém-nascidos brasileiros, uma maior discussão a respeito das causas desta contaminação torna-se difícil. Também não foram encontrados dados na literatura que reportassem a contaminação em outras amostras biológicas da população brasileira.

\section{CONCLUSÕES}

A praticidade da extração da matriz por hidrólise ácida e partição com solvente orgânico para determinação de PCBs em amostras de soro de cordão umbilical, combinada com GC- $\mu$ ECD e confirmada por GC-MS, foi demonstrada pelo presente estudo. O método demonstrou ser linear, preciso, exato e sensível, além de simples e de baixo custo, podendo ser utilizado em análises rotineiras para pequenas amostras de soro sanguíneo. Adicionalmente, a determinação dos compostos em amostras reais de soro de cordão umbilical demonstrou a conformidade do método. Os níveis de PCBs detectados nas 123 amostras analisadas demonstraram que a população pesquisada apresentou índices elevados de contaminação, comparando-se com estudos realizados em países da Europa e com os valores de referência estabelecidos pela ATSDR para a população americana.

Como este é o primeiro estudo a analisar e demonstrar diretamente os níveis de PCB em soro de cordão umbilical de recém-nascidos brasileiros, mais estudos são necessários para que se possam determinar as causas desta contaminação, bem como estabelecer valores de referência para a população brasileira.

\section{AGRADECIMENTOS}

Ao CNPq (processo 475787/2007-2), pelo aporte financeiro para a realização da pesquisa, e à CAPES (Edital Casadinhos). S. Mohr agradece à CAPES, pela bolsa de mestrado, I. Costabeber ao CNPq, pela bolsa de produtividade em pesquisa (PQ) e L. Soldatelli ao CNPq, pela bolsa de Iniciação Tecnológica Industrial (ITI).

\section{REFERÊNCIAS}

1.http://www.inchem.org/documents/ehc/ehc/ehc140.htm, acessada em Junho 2010 e Janeiro 2011.

2. Omaye, S. T.; Food and Nutritional Toxicology, CRC Press: Boca Ranton, 2004.

3. Colborn, T.; Meyers, J. P.; Dumanoski, D.; Nuestro Futuro Robado, Ecoespaña Editorial: Madrid, 1997.

4. Costabeber, I.; Emanuelli, T.; Bol. SBCTA 2003, 37, 1.

5. http://carlosminc.ning.com/profiles/blogs/minc-em-revista-15, acessada em Janeiro 2011.

6. Rylander, L.; Nilsson-Ehle, P.; Hagmar, L.; Chemosphere 2006, 62, 333.

7. Apostoli, P.; Magoni, M.; Bengonzi, R.; Carasi, S.; Indelicato, A.; Scarcella, C.; Donato, F.; Chemosphere 2005, 61, 413.

8. Turci, R.; Finozzi, E.; Catenacci, G.; Marinaccio, A.; Balducci, C.; Minoia, C.; Toxicol. Lett. 2006, 162, 250.

9. Nakamura, T.; Nakai, K.; Matsumura, T.; Suzuki, S.; Saito, Y.; Satoh, H.; Sci. Total Environ. 2008, 394, 39. 
10. Adenugba, A.; Khan, S. A.; Taylor-Robinson, S. D.; Cox, I. J.; Toledano, M. B.; Thillainayagam, A. V.; Bansi, D. S.; Thomas, H. C.; Gibson, R. W.; Beck, A. J.; Chemosphere 2009, 76, 841.

11. Agudo, A.; Goñi, F.; Etxeandia, A.; Vives, A.; Millán, E.; Lópes, R.; Amiano, P.; Ardanaz, E.; Barricarte, A.; Chirlaque, M. D.; Dorronsoro, M.; Jakszyn, P.; Larrañaga, N.; Martínez, C.; Navarro, C.; Rodríguez, L.; Sánchez, M. J.; Tormo, M. J.; González, C. A.; Environ. Res. 2009, 109,620 .

12. Zubero, M. B.; Ibarluzea, J. M.; Aurrekoetxea, J. J.; Rivera, J.; Parera, J.; Abad, E.; Goñi, F.; López, R.; Etxeandia, A.; Rodríguez, C.; Sáenz, J. R.; Chemosphere 2009, 76, 784.

13. Wittsiepe, J.; Fürst, P.; Schrey, P.; Lemm, F.; Kraft, M.; Eberwein, G.; Winneke, G.; Wilhelm, M.; Chemosphere 2007, 67, S286.

14. Zhao, G.; Xu, Y.; Li, W.; Han, G.; Ling, B.; Sci. Total Environ. 2007, 377, 179.

15. Cerná, M.; Malý, M.; Grabic, R.; Batáriová, A.; Smíd, J.; Benes, B.; Chemosphere 2008, 72, 1124.

16. Ayotte, P.; Muckle, G.; Jacobson, J. L.; Jacobson, S. W.; Dewailly, E.; Environ. Health Perspect. 2003, 111, 1253.

17. Jaraczewska, K.; Lulek, J.; Covaci, A.; Voorspoels, S.; Kaluba-Skotarczak, A.; Drews, K.; Schepens, P.; Sci. Total Environ. 2006, 372, 20.

18. Bengonzi, R.; Specchia, C.; Dinolfo, M.; Tomasi, C.; Palma, G. D.; Frusca, T.; Apostoli, P.; Chemosphere 2009, 76, 747.

19. Covaci, A.; Jorens, P.; Jacquemyn, Y.; Schepens, P.; Sci. Total Environ 2002, 298, 45 .

20. Tan, J.; Loganath, A.; Chong, Y. S.; Obbard, J. P.; Chemosphere 2009, $74,428$.

21. Goncharov, A.; Haase, R. F.; Santiago-Rivera, A.; Morse, G.; McCafferey, R.J.; Rej, R.; Carpenter, D.; Environ. Res. 2008, 106, 226.

22. Park, J. S.; Bergman, A.; Linderholm, L.; Athanasiadou, M.; Kocan, A.; Petrik, J.; Drobna, B.; Trnovec, T.; Charles, M. J.; Hertz-Picciotto, I.; Chemosphere 2008, 70, 1676.

23. Queiroz, S. C. N.; Collins, C. H.; Jardim, I. C. S. F.; Quim. Nova 2001, 24,68 .

24. Souza, M. H. L.; Elias, D. O.; Fundamentos da circulação extracorpórea, $2^{\text {a }}$ ed., Centro Editorial Alfa Rio: Rio de Janeiro, 2006.

25. Ramos, J. J.; Gómara, B.; Fernández, M. A.; Gonzáles, M. J.; J. Chromatogr., A 2007, 1152, 124.

26. Dmitrovic, J.; Chan, S. C.; Chan, S. H. Y.; Toxicol. Lett. 2002, 134, 253.

27. Gómara, B.; Ramos, L.; González, M. J.; J. Chromatogr., B: Anal. Technol. Biomed. Life Sci. 2002, 766, 279.

28. Basheer, C.; Lee, H. K.; Obbard, J. P.; J. Chromatogr., A 2004, 1022, 161.

29. López, R.; Goñi, F.; Etxandia, A.; Millán, E.; J. Chromatogr., B: Anal. Technol. Biomed. Life Sci. 2007, 846, 298.
30. Grimalt. J. O.; Carrizo, D.; Garí, M.; Font-Ribera, L.; Ribas-Fito, N.; Torrent, M.; Sunyer, J.; Environ. Res. 2010, 110, 244.

31. Carrizo, D.; Grimalt, J. O.; J. Chromatogr., A 2006, 1118, 271.

32. Porta, M.; Basea, M. B.; Benavides, F. G.; López, T.; Fernandez, E.; Marco, E.; Alguacil, J.; Grimalt, J. O.; Puigdomènech, E.; Environ. Res. 2008, 108, 370 .

33. Porta, M.; Jariod, M.; López, T.; Pumarega, J.; Puigdomènech, E.; Marco, E.; Malats, N.; Grimalt, J. O.; Real, F. X.; Environ. Int. 2009, 35,1080 .

34. Otero, R.; Santiago-Silva, M.; Grimalt, J. O.; J. Chromatogr., A 1997, $778,87$.

35. Sala, M.; Ribas-Fitó, N.; Cardo, E.; de Muga, M. E.; Marco, E.; Mazón, C.; Verdú, A.; Grimalt, J. O.; Sunyer, J.; Chemosphere 2001, 43, 895.

36. Ribani, M.; Bottoli, C. B. G.; Collins, C. H.; Jardim, I. C. S. F.; Melo, L. F. C.; Quim. Nova 2004, 27, 771.

37. Lanças, F. M.; Validação de métodos cromatográficos de análise, Rima: São Carlos, 2004.

38. Angulo, R.; Costabeber, I.; Gallego, M. C.; Serrano, S.; Jodral, M.; Livro de Resumos $1^{\text {st }}$ European Pesticide Residue Workshop, Alkmaar, Netherlands, 1996.

39. Agência Nacional de Vigilância Sanitária (ANVISA); Resolução RE $n^{o}$ 899 , de 29/05/2003.

40. Instituto Nacional de Metrologia, Normalização e Qualidade Industrial (INMETRO); Orientações sobre validação de métodos de ensaios químicos, DOQ-CGCRE-008, 2003.

41. Porta, M.; Grimalt, J. O.; Jariod, M.; Ruiz, L.; Marco, E.; López, T.; Malats, N.; Puigdomènech, E.; Zumeta, E.; Environ. Int. 2007, 33, 946.

42. Bucholski, K. A.; Begerow, J.; Winneke, G.; Dunemann, L.; J. Chromatogr., A 1996, 754, 479.

43. Lackmann, G. M.; Göen, T.; Töllner, U.; Schaller, K. H.; Angerer, J.; The Lancet 1996, 348, 1035.

44. http://www.cdc.gov/exposurereport/data_tables/, acessada em Janeiro 2011.

45. Santos, J. S.; Xavier, A. A. O.; Ries, E. F.; Costabeber, I.; Emanuelli, T.; Chemosphere 2006, 65, 1544 .

46. Heck, M. C.; Sifuentes dos Santos, J.; Bogusz Junior, S.; Costabeber, I.; Emanuelli, T.; Food Chem. 2007, 102, 288.

47. Costabeber, I.; Santos, J. S.; Xavier, A. A. O.; Weber, J.; Leães, F. L.; Bogusz Junior, S.; Emanuelli, T.; Food Chem. Toxicol. 2006, 44, 1.

48. http://extranet.agricultura.gov.br/sislegis/action/detalhaAto.do?method $=$ gravarAtoPDF $\&$ tipo $=I N M \&$ numeroAto $=00000008 \&$ seqAto $=000 \& \mathrm{~V}$ alorAno $=2010 \&$ orgao $=\mathrm{SDA} / \mathrm{MAPA} \& \operatorname{codTipo}=\&$ desItem $=\&$ desItemF $\mathrm{im}=$, acessada em Janeiro 2011. 